Urologe $2021 \cdot 60: 1141-1149$

https://doi.org/10.1007/s00120-021-01606-5

Angenommen: 7. Juli 2021

Online publiziert: 4 . August 2021

(C) Springer Medizin Verlag $\mathrm{GmbH}$, ein Teil von

Springer Nature 2021

\title{
Aktuelle Entwicklungen zur Digitalisierung
}

\section{Eine Analyse zu Qualität und Ökonomie in der Gesundheitsversorgung}

\author{
H. Dick $\cdot$ S. Doth ${ }^{1} \cdot$ C. Ernst ${ }^{1} \cdot$ S. Fischer ${ }^{1} \cdot$ M. Holderried ${ }^{1,2}$ \\ 'Institut Health Care \& Public Management, Lehrstuhl für Ökonomik und Management sozialer \\ Dienstleistungen (530B), Universität Hohenheim, Stuttgart, Deutschland \\ ${ }^{2}$ Zentralbereich Medizin: Struktur-, Prozess- und Qualitätsmanagement, Universitätsklinikum Tübingen, \\ Tübingen, Deutschland
}

\section{Zusammenfassung}

\section{In diesem Beitrag}

- Digitalisierung, Ökonomie und Qualität im Gesundheitswesen

- Gutachten des Sachverständigenrates zu Digitalisierung im Gesundheitswesen

- Ökonomische und institutionelle Rahmenbedingungen

- Ausgewählte Anwendungsbereiche digitaler Innovationen

- Strukturierte Befundung

- Anwendung strukturierter Befundung im Kontext der Urologie

- Telemedizin in der Urologie

- Aktueller Stand der Forschung zu Effektivität und Effizienz von telemedizinischen Anwendungen in der Urologie

Hintergrund: Im deutschen Gesundheitssystem und damit auch im Fachgebiet der Urologie gewinnen ökonomische Rahmenbedingungen zunehmend an Bedeutung und parallel dazu werden digitale Anwendungen vermehrt eingesetzt.

Fragestellung: Die Fragestellung betrifft die gesundheitsökonomische Auseinandersetzung mit den Rahmenbedingungen der Digitalisierung im deutschen Gesundheitssystem sowie ausgewählter Anwendungsbereiche in der Urologie. Material und Methoden: Das Gutachten des Sachverständigenrates zur Begutachtung der Entwicklung im Gesundheitswesen (SVR) wird analysiert und eine systematische Literaturanalyse zum Einsatz der strukturierten Befundung und Analyse ausgewählter Literatur zu telemedizinischen Anwendungen in der Urologie unter gesundheitsökonomischen Gesichtspunkten durchgeführt.

Ergebnisse: Als zentrale Hemmnisse bei der Digitalisierung des deutschen Gesundheitswesens identifiziert der SVR dessen Regulierung und Komplexität sowie den Umgang mit Datenschutz und -sicherheit. Der Einsatz strukturierter Befundung kann Qualität, Effektivität und Effizienz der Befundung in der Urologie steigern. Im Hinblick auf die Kosten können signifikante Einsparungen mit zunehmender Digitalisierung in der Medizin realisiert werden.

Schlussfolgerungen: Aus medizinischer und gesundheitsökonomischer Perspektive besteht bei der Ausgestaltung von Rahmenbedingungen für digitale Anwendungen im deutschen Gesundheitssystem hinsichtlich der Informationssicherheit und des Datenschutzes weiterer Gestaltungsbedarf. Bei zielgerichtetem Einsatz von digitalen Anwendungen wie der strukturierten Befundung und der Telemedizin können optimale Voraussetzungen für den zunehmenden Einsatz von künstlicher Intelligenz im Fachgebiet der Urologie geschaffen werden.

\section{Schlüsselwörter}

EHealth · Gesundheitsökonomie · Telemedizin · Elektronische Patientenakte · QALY

Mit dem vorliegenden Artikel wird ein gesundheitsökonomischer Überblick zum aktuellen Stand ausgewählter Aspekte der Digitalisierung in einzelnen Anwendungsgebieten im deutschen Gesundheitswesen vermittelt, wobei besonders auf die Urologie eingegangen wird. Unter Qualitäts- und Kostenaspekten wird eine gesundheitsökonomische Einschätzung im Hinblick auf Effektivität und Effizienz vorgenommen. Auf Aspekte zu strukturierter Befundung und telemedizinischen Anwendungen in der Urologie wird detaillierter eingegangen.

\section{Digitalisierung, Ökonomie und Qualität im Gesundheitswesen}

Durch das Auftreten der COVID-19-Pandemie („'coronavirus disease 2019") hat sich 


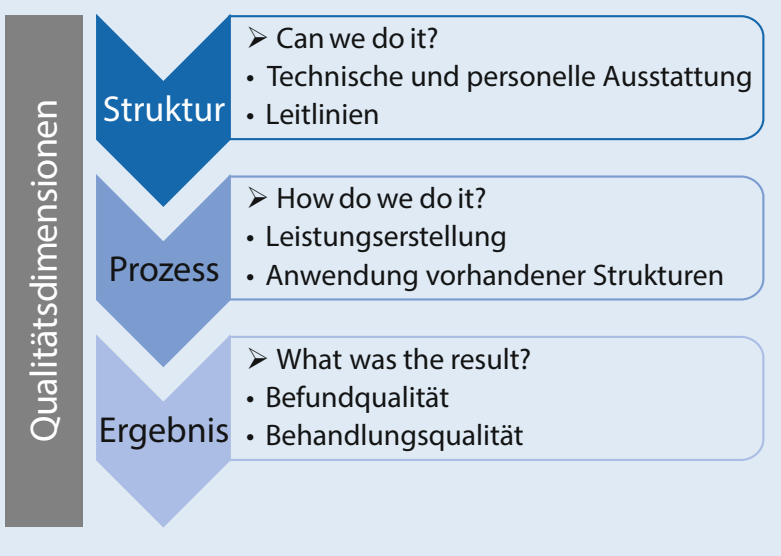

das ambulante und stationäre Leistungsgeschehen in der Gesundheitsversorgung maßgeblich verändert. Der Einsatz von telemedizinischen Anwendungen hat an Bedeutung gewonnen, um eine Gesundheitsversorgung zu gewährleisten, welche Patienten und Ärzte vor dem Risiko schützen, sich einer COVID-19-Infektion auszusetzen [28]. So wurden in Deutschland bspw. die rechtlichen Regelungen zur Durchführung und zur Vergütung von Videosprechstunden angepasst und ausgeweitet. Langfristige Zielsetzung sollte es demnach sein, die durch die COVID-19-Pandemie angestoßene Entwicklung zu mehr Digitalisierung dauerhaft in das Gesundheitssystem zu integrieren und neue Lösungsansätze für die medizinische Versorgung zu implementieren [20].

\section{\) Innerhalb des Gesundheitswe- sens wird die Ökonomie häufig als ein restriktives Element angesehen}

Innerhalb des Gesundheitswesens wird die Ökonomie häufig als ein restriktives Element angesehen. Eine mögliche Ursache für diese Sichtweise kann in den unterschiedlichen Perspektiven von Gesundheitsberufen einerseits und Ökonomen andererseits begründet liegen. Der Fokus des medizinischen Fachpersonals liegt auf der möglichst optimalen Behandlung von einzelnen Patienten. In der Ökonomie liegt der Fokus neben einer effektiven Versorgung auch auf einer effizienten Ressourcenverwendung, da diese nur begrenzt zur Verfügung stehen [4]. Die Tragweite hieraus entstehender Abwägungsentscheidungen (sog. "trade-off") hat sich im Rahmen der COVID-19-Pandemie bspw. in der intensivmedizinischen Versorgung oder Verteilung des zunächst knappen Impfstoffs eindrucksvoll verdeutlicht. Es verwundert daher nicht, dass aus diesen unterschiedlichen Perspektiven ein gewisses Konfliktpotential zwischen Medizin und Pflege einerseits sowie Ökonomie andererseits entstehen kann. Vor dem Hintergrund des fortschreitenden demographischen Wandels, der damit verbundenen Alterung der Gesellschaft, den neuen technischen Möglichkeiten in der Medizin sowie der zunehmenden Prävalenz von in ihrer Behandlung ressourcenaufwändigen chronischer Erkrankungen erscheint ein Abklingen dieses Konflikts eher unwahrscheinlich. In der Verwendung digitaler Gesundheitsprodukte und -dienstleistungen kann dabei eine Möglichkeit bestehen, Aspekte der Ressourceneffizienz und Behandlungsbzw. Versorgungsqualität simultan im Blick zu behalten.

Der Einsatz von digitalen Anwendungen verfügt über das Potenzial als effizientes, zugängliches und kosteneffektives Modell für die Bereitstellung von Gesundheitsleistungen akzeptiert zu werden [15]. Insbesondere im Fachgebiet der Urologie verfügen telemedizinische Anwendungen über ein wesentliches Potenzial, die Qualität und die Effizienz in der urologischen Patientenversorgung zu erhöhen sowie örtliche und zeitliche Grenzen zu überwinden [25].

Im Gesundheitswesen wird Qualität häufig anhand der von Avedis Donabedian definierten Qualitätsdimensionen der Struktur-, Prozess- und Ergebnisqualität erhoben. Die Strukturqualität beschreibt Elemente der Infrastruktur, also Ausstattung und Voraussetzungen für die Gesundheitsversorgung. Innerhalb der Prozessqualität werden die Vorgänge und Prozessabläufe selbst analysiert. In der Ergebnisqualität werden schließlich die Outcomes der Prozesse evaluiert. Somit ist ein Zusammenspiel der drei Ebenen zu erkennen, da eine hohe Prozessqualität eine gute Strukturqualität erfordert und eine gute Ergebnisqualität nur erreicht werden kann, wenn die vorgelagerte Strukturund Prozessqualität gesichert ist. Dieser Zusammenhang ist in $\mathbf{A b b} .1$ dargestellt [23]. Sehr oft besteht dabei in der Medizin zwischen Qualität und Ökonomie leider eine konfliktäre Beziehung. Verbesserungen in der Patientenversorgung verursachen häufig höhere Kosten als der Status quo [13]. Allerdings sind auch komplementäre Beziehungen zwischen Qualität und Ökonomie denkbar, d.h. eine zumindest gleichbleibende oder gar verbesserte Qualität geht mit geringeren Kosten einher [2].

\section{Gutachten des Sachverständi- genrates zu Digitalisierung im Gesundheitswesen}

Der Sachverständigenrat zur Begutachtung der Entwicklung im Gesundheitswesen (SVR) hat sich in seinem Gutachten von 2021 "Digitalisierung für Gesundheit" mit den Chancen und Risiken des Einsatzes digitaler Innovationen in Deutschland auseinandergesetzt [8]. Dabei hat der SVR neben den ökonomischen Rahmenbedingungen und aktuellen wie potenziellen Anwendungsbereichen auch den Zusammenhang mit dem gerade im Gesundheitswesen untrennbaren Aspekt des Datenschutzes beleuchtet. Der SVR sieht in der gegenwärtigen rechtlichen Handhabung ein Hindernis in der Einführung digitaler Innovationen und empfiehlt neben rechtlichen Rahmenbedingungen v.a. das Setzen technischer Standards, die es ermöglichen, anbieter- und sektorübergreifend qualitätsgesicherte Patientendaten im Sinne des Patientenwohls zu verwenden. Auf Ebene des einzelnen Patienten bestünden so Chancen für "eine verbesserte individuelle Behandlung" und auf Ebene der Allgemeinheit eine Chance 
Hier steht eine Anzeige.

黑 Springer 
zu „einer bedarfsgerechten Weiterentwicklung der Gesundheitsversorgung" [8, 14].

\section{Ökonomische und institutionelle Rahmenbedingungen}

Nach der Begutachtung ökonomischer und institutioneller Rahmenbedingungen des deutschen Gesundheitswesens identifiziert der SVR dessen hohe Komplexität und starke Regulierung als mögliche Hindernisse bei der Adaption digitaler Technologien. Die starke Regulierung leitet sich aus den Eigenschaften von Gesundheitsmärkten $a b$ :

\section{I) Der SVR empfiehlt neben rechtlichen Rahmenbedingungen \\ v. a. das Setzen technischer Standards}

Gesundheitsmärkte sind durch ein hohes $\mathrm{Ma}$ an Expertenwissen und ein erhebliches Informationsgefälle zwischen Leistungsanbietern und -nachfragern (Patienten) gekennzeichnet (sog. asymmetrische Informationsverteilung). Einige Ökonomen bezeichnen deshalb medizinische Diagnostik und Therapie als sog. „Vertrauensgüter" ("credence good"), denn der Patient kann deren Qualität oft selbst nach der Behandlung nicht abschließend beurteilen. Der Gesundheitsmarkt zählt zu den größten Vertrauensgütermärkten der meisten Volkswirtschaften mit Eigenschaften wie das Vorhandensein von ausgewiesenen Experten und bestehenden Preisregulierungen [19]. Des Weiteren wird das "Gut" Gesundheit durch das Vorliegen „externer Effekte“ charakterisiert: Von der Nachfrage einiger Gesundheitsgüter (bspw. durch die aktuellen Coronaimpfungen) profitiert nicht nur der Nachfragende selbst, sondern insbesondere auch die Gesellschaft. Folge dieser Eigenschaften wäre eine unzureichende Nachfrage nach Gesundheitsdienstleistungen im Verhältnis zum gesellschaftlichen Optimum. Man spricht dabei von "Marktversagenstatbeständen", welche ein Eingreifen des Staates rechtfertigen [4].

Die hohe Komplexität ergibt sich hingegen v.a. aus der historisch gewachsenen Trennung von Verantwortlichkeiten bei der Gesundheitsversorgung im Rah- men der Selbstverwaltung des föderalen Systems sowie sektorspezifischen Regelungen. Insbesondere mit den Besonderheiten des deutschen Gesundheitssystems weniger vertrauten Anbietern digitaler Gesundheitsleistungen stellt die Kombination aus deutschem Datenschutzrecht, fragmentierter Organisation und starker Regulierung eine große Herausforderung beim Marktzutritt dar [8].

Auch aus der ökonomischen Charakteristik digitaler Produkte und Dienstleistungen lassen sich Handlungsempfehlungen ableiten. Diese sind in der Regel durch eine typische Kostenstruktur gekennzeichnet: Anfänglich fallen bei der Entwicklung von Hard- und Softwarelösungen sowie beim Aufbau einer digitalen Infrastruktur hohe Kosten an. Die (laufenden) Betriebskosten sind im Verhältnis dazu allerdings gering. Im Hinblick auf die Stückkosten (z.B. pro Therapie) sinken die hohen Investitionskosten mit der Anwendungszahl (sog. „economies of scale").

Bei digitalen Plattformen lässt sich ein ähnlicher Effekt im Hinblick auf die Nutzerzahl beobachten: Mit steigender Nutzerzahl wird die Plattform für Akteure außerhalb der Plattform attraktiver. Gleichzeitig steigt der Nutzen für die im Netzwerk vorhandenen Nutzer an (sog. "network economics“). Zuletzt werden digitale (Gesundheits)produkte häufig bewusst als Produkt- bzw. Leistungsverbund innerhalb der jeweiligen technischen Infrastruktur angeboten. Folgen dieser Charakteristik sind häufig eine erhebliche Marktkonzentration bis zu Entstehung von natürlichen Monopolen und ein hoher Aufwand beim Anbieterwechsel (sog. Lock-in-Effekt). Beide Umstände erschweren den Wettbewerb auf dem entsprechenden Markt und sind in der Regel mit geringer Innovationstätigkeit und abnehmender Produkt- und Dienstleistungsqualität gekennzeichnet [30]. Von besonderer Bedeutung ist dabei die Interoperabilität, d.h. die Kompatibilität unterschiedlicher informations- und kommunikationstechnischer Systeme: Um geeignete wettbewerbliche Voraussetzungen im deutschen Gesundheitsmarkt zu schaffen, ist es laut SVR notwendig, auf technischer Ebene Standards zu etablieren, welche insbesondere eine anbieter- und sektorübergreifende Vernetzung und Kommunikation ermöglichen.

\section{Ausgewählte Anwendungsberei- che digitaler Innovationen}

Bei der Analyse der Anwendungsbereiche digitaler Innovationen konzentriert sich der SVR auf digitale Gesundheitsanwendungen (DiGA) und die geplante Einführung der elektronischen Patientenakte (ePA). Die bisher angedachte Form der Einführung der ePA als mehrfach zustimmungspflichtig birgt laut SVR das Risiko, dass der damit verbundene Aufwand Patienten wie Leistungserbringer von deren Verwendung abhalten könnte. Der SVR empfiehlt eine Widerspruchslösung, wenngleich er auf die Notwendigkeit zur Aufklärung des individuellen und kollektiven Nutzens sowie der die Datensicherheit betreffenden Risiken durch Institutionen der Gesundheitsversorgung wie bspw. der Krankenkassen hinweist [14].

\section{॥ Bei Analyse der Anwendungs- bereiche digitaler Innovationen konzentriert sich der SVR auf DiGA und ePA}

Für die Aufnahme von DiGA in das Leistungsspektrum der Krankenkassen empfiehlt der SVR ein Nutzenbewertungsverfahren nach dem Vorbild der Bewertung von Arzneimitteln und Medizinprodukten. Dabei weist der SVR auf die entscheidungsunterstützende Methodik gesundheitsökonomischer Evaluationen hin. So können im Rahmen von Kosten-NutzwertAnalysen Effekte auf Mortalität und Morbidität häufig ausgedrückt in Form von QALY ("quality adjusted life years") in Relation zu den erwarteten Kosten gesetzt werden. Um die oben beschriebenen Lock-in-Effekte zu vermeiden bzw. den Wettbewerb im Bereich DiGA zu sichern, wird erneut auf das Setzen technischer Standards und die Sicherstellung von Interoperabilität der verwendeten IT-Systeme verwiesen [8].

\section{Strukturierte Befundung}

Die Digitalisierung im Gesundheitswesen findet auch Anwendung bei der Erstellung von Befunden. Unter dem Begriff "structured reporting " („strukturierte Befundung ") 


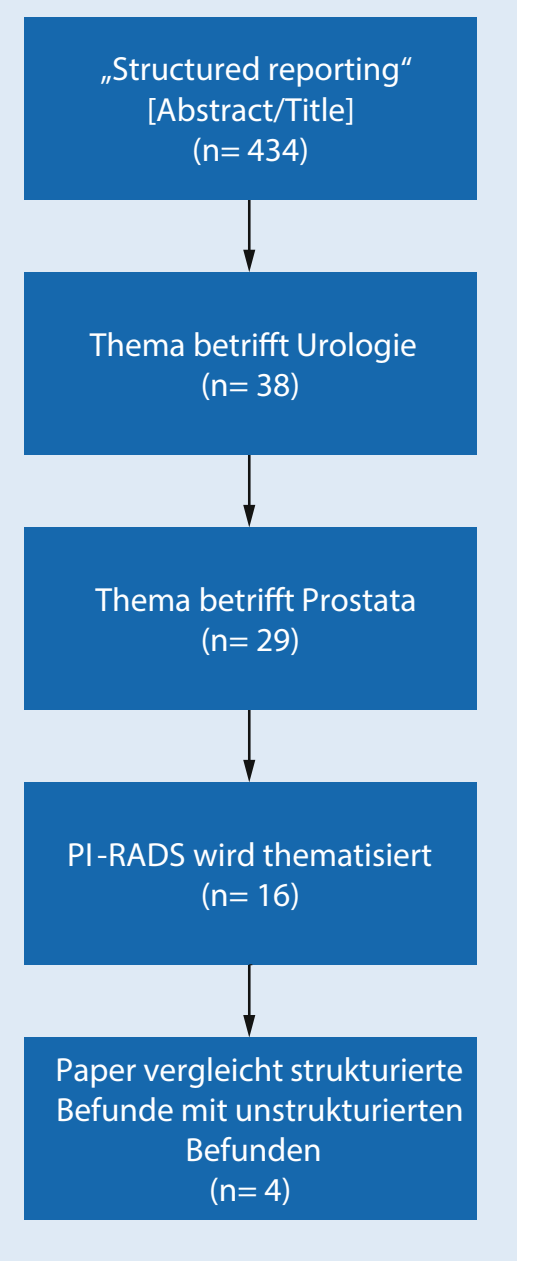

Abb. $2 \Delta$ Systematische Literaturanalyse zur strukturierten Befundung in der Urologie ( $P I-$ $R A D S$,prostate imaging reporting and data systems")

werden Befunde verstanden, welche einer standardisierten Gliederung folgen und Formulierungen zur Beschreibung von Beobachtungen enthalten. So können befundende Ärzte durch die Auswahl aus einem Drop-down-Menü Beobachtungen dokumentieren und aus Formulierungen, welche im System hinterlegt sind, ergibt sich letztlich ein Befund als Fließtext [17].

Der Einsatz von strukturierter Befundung soll dabei helfen, die Effizienz bei der Erstellung und der Kommunikation zu erhöhen, um letztlich eine erhöhte Berichtsqualität sicherzustellen [5]. Durch einheitliche Klassifikationssysteme, wie diese beispielsweise für Brustuntersuchungen (BIRADS [„, breast imaging-reporting and data systems"]), für Leberuntersuchungen (나RADS) und für Untersuchungen der Pros- tata (PI-RADS) bestehen, wird die Eindeutigkeit von Befunden bereits in mehreren Fachrichtungen der Medizin sichergestellt [1]. Darüber hinaus bietet eine strukturierte Befundung optimale Voraussetzungen für die weitere Integration von maschinellen Lernprozessen bzw. der künstlichen Intelligenz (KI) in die Gesundheitsversorgung [22].

\section{Anwendung strukturierter Befundung im Kontext der Urologie}

Um die Befundung von MRT der Prostata anhand der S3-Leitlinie für Prostatakarzinome einheitlich zu erstellen, wurde das strukturierte Befundungsschema PI-RADS von der Expertengruppe der European Society of Urogenital Radiology (ESUR) entwickelt [24].

Bei Nutzung strukturierter Berichtsvorlagen werden einheitliche Klassifizierungen - wie mithilfe von PI-RADS definiert häufiger eingehalten [27]. Dadurch wird die interdisziplinäre Kommunikation zwischen Ärzten verbessert, die Qualität der Untersuchungsbefunde gesichert und die Durchführung medizinischer Studien erleichtert [9]. Der Effekt des Einsatzes strukturierter Befundung unter Verwendung der PI-RADS-Klassifikation gegenüber konventioneller Freitextbefundung ist allerdings bislang nur wenig erforscht. Die in - Abb. 2 dargestellte systematische Literaturanalyse in der Datenbank PubMed mit dem Suchbegriff "structured reporting" in Abstract oder Titel ergibt für den Suchzeitraum von 2010 bis Juni 2021 insgesamt 434 Ergebnisse. Nach Sichtung aller Arbeiten wurden 38 Arbeiten mit thematischem Bezug zur Urologie identifiziert. Hiervon ist in 29 Arbeiten der Themenbezug zur Prostata erkennbar. Das Klassifikationssystem PI-RADS wird von 16 Arbeiten thematisiert. Lediglich in 4 Artikeln [16, 27, 33, 34] werden strukturierte mit unstrukturierten Befunden vergleichend analysiert (s. Appendix 1).

Magnetta et al. [16] dokumentieren in einer objektiven Untersuchung eine höhere Vollständigkeit relevanter Inhalte bei Verwendung einer strukturierten Berichtsvorlage. Auch unter subjektiven Kriterien konnte verbesserte Konsistenz, Vollständigkeit, Klarheit und erhöhte wahrgenommene klinische Wertigkeit bei Verwendung strukturierter Berichtsvorlage beobachtet werden. Zudem berichten Radiologen von einer empfundenen schnelleren Befundung bei der Verwendung von strukturierten Berichtsvorlagen. In anderen Fachbereichen konnte diese Zeitersparnis beim Vergleich von strukturierter Befundung gegenüber der Freitextbefundung bereits empirisch belegt werden [7].

\section{॥ Strukturierte Befunde verbessern die Kommunikation von Radiologen mit weiterbehandelnden Urologen}

Durch den Einsatz strukturierter Befunde von Radiologen wurde die Kommunikation mit weiterbehandeInden Urologen verbessert. Radiologen berichten von keinen Barrieren in der Verwendung von strukturierter Befundung [16]. Weitere Vorteile ergeben sich in der genaueren Identifikation von beschriebenen Befunden, einer höheren wahrgenommenen Qualität der Berichte und der Möglichkeit, fundierte Rückschlüsse für die Weiterbehandlung zu ziehen ([33]; - Tab. 1).

Die Analyse von Silveria et al. (2015) zeigt eine steigende Zufriedenheit mit Befunden, welche IT-gestützt erstellt wurden. Radiologen präferieren dabei freiere Befundvorlagen, um mehr Flexibilität zu haben, während Urologen mehr Standardisierung für wichtig halten. Hier sollte bei strukturierten Berichtsvorlagen ein Mittelweg gewählt werden [26].

Die technische Umsetzung strukturierter Befundung in ein Programm, welches auch die PI-RADS-Systematik beinhaltet, wurde im Jahr 2016 von Mata et al. [18] beschrieben. Wenn strukturierte Befundvorlagen korrekt implementiert sind, kann die Befundqualität gesteigert werden. In Folge der stärkeren Beachtung von standardisierten Expertenrichtlinien wie PI-RADS kann im besten Fall eine verbesserte Therapieentscheidung für die Patienten resultieren [26].

Insgesamt ist festzuhalten, dass strukturierte Befundung in der Urologie mit dokumentierten Qualitätsverbesserungen einherzugehen scheint. Die Verwendung strukturierter Befundung kann die Strukturqualität erhöhen. Durch Zeiteinsparungen und Qualitätsverbesserungen bei der 
Tab. 1 Übersicht ausgewählter Arbeiten zu strukturierter Befundung

\begin{tabular}{|c|c|c|c|c|c|}
\hline Autoren & Jahr & Titel & Zielsetzung & Methodik & Ergebnis \\
\hline $\begin{array}{l}\text { Magnetta } \\
\text { et al. }\end{array}$ & 2018 & $\begin{array}{l}\text { Evidence-Based Reporting: } \\
\text { A Method to Optimize Pros- } \\
\text { tate MRI Communications } \\
\text { With Referring Physicians }\end{array}$ & $\begin{array}{l}\text { Entwicklung einer evidenz- } \\
\text { basierten Methode zur Opti- } \\
\text { mierung von Prostata-MRT- } \\
\text { Berichten }\end{array}$ & $\begin{array}{l}\text { Entwicklung einer Berichts- } \\
\text { vorlage unter Verwendung } \\
\text { von PI-RADS Komponenten, } \\
\text { Vergleich von } 40 \text { Berichten } \\
\text { jeweils vor und nach der } \\
\text { Implementierung }\end{array}$ & $\begin{array}{l}\text { Strukturierte Berichte führen } \\
\text { zu besserer Kommunikation } \\
\text { mit überweisenden Ärzten }\end{array}$ \\
\hline $\begin{array}{l}\text { Shaish } \\
\text { et al. }\end{array}$ & 2018 & $\begin{array}{l}\text { Impact of a Structured Re- } \\
\text { porting Template on Adhe- } \\
\text { rence to Prostate Imaging } \\
\text { Reporting and Data Sys- } \\
\text { tem Version } 2 \text { and on the } \\
\text { Diagnostic Performance of } \\
\text { Prostate MRI for Clinically } \\
\text { Significant Prostate Cancer }\end{array}$ & $\begin{array}{l}\text { Bewertung der Auswirkun- } \\
\text { gen einer strukturierten } \\
\text { Berichtsvorlage auf die Ein- } \\
\text { haltung der PI-RADS-Syste- } \\
\text { matik (Version 2) und auf die } \\
\text { diagnostische Wertigkeit des } \\
\text { Prostata-MRT zur Erkennung } \\
\text { von Prostatakrebs }\end{array}$ & $\begin{array}{l}\text { Untersuchung von Radiolo- } \\
\text { giebefunden vor und nach } \\
\text { Aktualisierung der Berichts- } \\
\text { vorlage bei } 202 \text { Patienten }\end{array}$ & $\begin{array}{l}\text { Strukturierte Vorlage mit } \\
\text { Drop-down-Menüs, verbes- } \\
\text { sert Einhaltung der PI-RADS } \\
\text { und erhöht diagnostische } \\
\text { Leistung von Prostata-MRT }\end{array}$ \\
\hline $\begin{array}{l}\text { Wetterauer } \\
\text { et al. }\end{array}$ & 2019 & $\begin{array}{l}\text { Structured reporting of pros- } \\
\text { tate magnetic resonance } \\
\text { imaging has the potential } \\
\text { to improve interdisciplinary } \\
\text { communication }\end{array}$ & $\begin{array}{l}\text { Untersuchung, ob strukturier- } \\
\text { te Berichte der Prostata-MRT } \\
\text { die interdisziplinäre Kommu- } \\
\text { nikation verbessern können }\end{array}$ & $\begin{array}{l}\text { Vergleich von je } 50 \text { unstruk- } \\
\text { turierten und strukturierten } \\
\text { Berichten durch Urologen }\end{array}$ & $\begin{array}{l}\text { Strukturierte Berichterstat- } \\
\text { tung hat das Potenzial die } \\
\text { interdisziplinäre Kommuni- } \\
\text { kation zu verbessern }\end{array}$ \\
\hline $\begin{array}{l}\text { Wetterauer } \\
\text { et al. }\end{array}$ & 2019 & $\begin{array}{l}\text { Novices in MRI-targeted } \\
\text { prostate biopsy benefit from } \\
\text { structured reporting of MRI } \\
\text { findings }\end{array}$ & $\begin{array}{l}\text { Untersuchung ob struktu- } \\
\text { rierte Berichte der Prostata- } \\
\text { MRT besser geeignet sind als } \\
\text { nichtstrukturierte Berichte, } \\
\text { um die genaue Beurteilung } \\
\text { einer Prostatakrebsläsion } \\
\text { durch unerfahrene Ärzte zu } \\
\text { fördern }\end{array}$ & $\begin{array}{l}\text { Jeweils } 50 \text { Berichte wurden } \\
\text { von unerfahrenen Ärzte } \\
\text { analysiert und die Qualität } \\
\text { anhand eines Fragebogens } \\
\text { verglichen }\end{array}$ & $\begin{array}{l}\text { Strukturierte Berichterstat- } \\
\text { tung der Prostata-MRT als } \\
\text { Informationsinstrument } \\
\text { werden bevorzugt, struktu- } \\
\text { rierte Berichte können den } \\
\text { Lernprozess fördern }\end{array}$ \\
\hline
\end{tabular}

Befundung wird die Prozessqualität erhöht und es besteht die Möglichkeit, die Kosteneffizienz des gesamten Behandlungsprozesses zu steigern. Als Resultat dieser beiden Qualitätsverbesserungen kann in der Ergebnisqualität neben vollständigen, korrekten und einheitlich verständlichen Befunden auch eine verbesserte $\mathrm{Pa}$ tientenversorgung resultieren. Innerhalb der Urologie besteht noch großer Forschungsbedarf hinsichtlich der Kosteneffizienz dieses Instruments. Gegenwärtig konnten keine Kosteneffektivitätsstudien zu dieser Thematik im Gebiet der Urologie identifiziert werden, daher gilt es, dies in künftigen Studien vermehrt zu adressieren. Entsprechend der Analyse des Einsatzes strukturierter Befundung in angrenzenden Fachgebieten ist davon auszugehen, dass aufgrund des geschilderten Economies-of-scale-Effekts, Potenzial zur Steigerung der Kosteneffizienz besteht [3].

\section{Telemedizin in der Urologie}

Zu den vielfältigen Nutzenpotentialen, die mit dem Einsatz von telemedizinischen Anwendungen verbunden sind, zählen der gesicherte Datenaustausch und die Kommunikation zwischen Patienten, Leistungserbringern und Leistungsträgern über räumliche Distanzen hinweg. Weitere positive Aspekte ergeben sich in der Sicherung der Diagnosegüte, der Erhöhung der Patientenzufriedenheit und bei der Unterstützung einer adäquaten Versorgung von Patienten in unterversorgten Regionen [11, 32]. Weiterhin können telemedizinische Anwendungen zu einer gesteigerten Therapieadhärenz der Patienten beitragen und damit eine Optimierung der gesundheitlichen Versorgung zu ermöglichen sowie die Bindung der Patienten an Klinik und Praxis erhöhen [29]. Hervorzuheben ist dabei, dass die Patienten über alle Altersgruppen hinweg eine steigende Affinität zu DiGA aufweisen und auch die Chancen und Potenziale von eHealth-Anwendungen fachbereichsübergreifend zunehmend positiv bewerten [10, 11].

\section{Aktueller Stand der Forschung zu Effektivität und Effizienz von telemedizinischen Anwendungen in der Urologie}

Trotz dieser vielfältigen Nutzenpotentiale der telemedizinischen Anwendungen ist der aktuelle Stand der Literatur zu gesundheitsökonomischen Evaluationsstudien derzeit als deutlich ausbaufähig anzusehen [21].

Im Hinblick auf Qualität, Effektivität und Effizienz in der Patientenversorgung konnten im Rahmen einer Literaturanalyse ausgewählte Studienergebnisse identifiziert werden.

Viers et al. [31] konnten in einer randomisiert kontrollierten Studie mit 55 Prostatektomiepatienten zeigen, dass die Durchführung von Videovisiten zu einer vergleichbaren Effizienz und Patientenzufriedenheit führen, wie dies bei herkömmlichen Praxisbesuchen der Fall ist. Aus der Patientenperspektive konnten Kosten eingespart werden, indem sich Reisezeit (0 vs. $95 \mathrm{~min}$ ) und -kosten (0 US\$ vs. 48 US\$) sowie die Anzahl an verpass- 
Hier steht eine Anzeige.

黑 Springer 
ten Arbeitstagen (0 vs. 1 Tag) pro Patient signifikant reduzierten.

Chu et al. [6] präsentieren Ergebnisse zur Einführung einer urologischen Telemedizinklinik in ländlichen Regionen der USA. Die Patientenzufriedenheit der telemedizinisch behandelten Patienten war in $95 \%$ der Fälle „sehr gut" bis ausgezeichnet und $97 \%$ der behandelten Patienten würden die urologische Telemedizinklinik weiterempfehlen. Neben der hohen Patientenzufriedenheit konnten die behandelten Patienten durchschnittlich Reisekosten in Höhe von 67 US\$ und Reisezeit in Höhe von 290 min einsparen. Die durchschnittlich vermiedenen Opportunitätskosten, repräsentiert durch ausgefallene Arbeitszeit des Patienten betrugen 126 US\$ pro Person.

Jones et al. [15] führten eine randomisiert kontrollierte Studie mit 195 Patientinnen durch, in welcher eine telefonische Sprechstunde mit einer regulären Vor-Ort-Sprechstunde hinsichtlich Patientenerlebnis und Kosten verglichen wurde. Die Ergebnisse der Studie zeigen keine signifikanten Unterschiede hinsichtlich des kurzfristigen Patientenerlebnisses. Zu konstatieren ist, dass signifikante Unterschiede hinsichtlich einer verbesserten Kommunikation, reduzierter Konsultationszeit $(10,9$ vs. $25,9 \mathrm{~min})$ und geringerer Konsultationskosten $(31,75$ vs. $72,17 £$ ) bestehen. Die telefonische Beratung im Vergleich zur Standardversorgung war mit höheren Wiedervorstellungen in der Praxis verbunden, wobei die damit einhergehende durchschnittliche Kostendifferenz von 38,04 $£$ zwischen der Interventions- und Kontrollgruppe nicht signifikant ist.

Zholudev et al. [35] führten einen Kostenvergleich zwischen 300 Patienten einer teleurologischen Klinik und $100 \mathrm{~Pa}$ tienten einer regulären Klinik durch. Im Ergebnis zeigt die Studie, dass die reguläre Behandlung mit einer durchschnittlich höheren Behandlungszeit (266 vs. $70 \mathrm{~min}$, $p<0,001)$ und mit durchschnittlich höheren Kosten pro Sitzung $(135,02$ vs. $10,95$ US\$, $p<0,001)$ einhergeht. Als maßgeblicher Faktor für die Kosteneinsparungen in Höhe von 124,07 US\$ bei der telemedizinischen Behandlung sind die Reisekosten und die Opportunitätskosten des Patienten sowie die Kosten für das Klinikpersonal zu nennen.
Eine weitere Studie von Holten-Rossing et al. [12] untersuchte den Nutzen von Telekonsultationen im Rahmen der Echtzeitbefundung von urologischen Tumoroperationen, bei denen eine Referenzpathologie benötigt wurde. Die Autoren stellen mit der Studie fest, dass die Anwendung von Telekonsultationen im untersuchten Kontext mit reduzierten Kosten für Fracht und Transport einhergeht und zeitnahe Patientenüberweisungen erfolgen können. Ferner ist bei einer sorgfältigen Selektion der Gewebeproben die diagnostische Sicherheit nicht beeinträchtigt.

\section{I) Durch telemedizinische Anwendungen können in der Urologie Kosten eingespart werden}

In einer zusammenfassenden Betrachtung der Studienergebnisse ist zu konstatieren, dass alle gesichteten Studien aus medizinischer wie ökonomischer Sicht ein einheitliches und optimistisches Bild hinsichtlich Qualität, Effektivität und Effizienz von telemedizinischen Anwendungen ergeben. Bei gleichbleibender oder gar verbesserter Qualität konnten durch telemedizinische Anwendungen in der Urologie Kosten eingespart werden. Die Studienergebnisse lassen zumindest für dieses Anwendungsfeld schlussfolgern, dass Qualität und Ökonomie in keinem konfliktären, sondern auch in einem komplementären Verhältnis zueinanderstehen können. Damit profundere Aussagen hinsichtlich Qualität, Effektivität und Effizienz von telemedizinischen Anwendungen getroffen werden können, sind in Zukunft weitere gesundheitsökonomische Evaluationsstudien mit einem langfristigen Betrachtungszeitraum zu entwickeln und hinsichtlich der Übertragbarkeit auf das deutsche Gesundheitssystem zu prüfen [21].

\section{Fazit für die Praxis}

- Digitale Anwendungen ermöglichen es, Aspekte der Ressourceneffizienz und Behandlungs- bzw. Versorgungsqualität simultan im Blick zu behalten.

- Von besonderer Bedeutung sind die Interoperabilität von IT-Systemen sowie ein neues Verständnis von Datensicherheit zur anbieter- und sektorübergreifenden Vernetzung.

- Die Einführung der elektronischen Patientenakte kann als Schlüsselelement zu mehr Digitalisierung im Gesundheitswesen verstanden werden.

- Die Verwendung strukturierter Befundung kann die Strukturqualität erhöhen. Durch Zeiteinsparungen bei der Befundung wird die Prozessqualität erhöht. In der Ergebnisqualität kann eine verbesserte Patientenversorgung resultieren.

- Der Einsatz von telemedizinischen Anwendungen verfügt über das Potenzial, Qualität, Effektivität und Effizienz in der Patientenversorgung zu erhöhen.

\section{Korrespondenzadresse}

\section{Prof. Dr. C. Ernst}

Institut Health Care \& Public Management, Lehrstuhl für Ökonomik und Management sozialer Dienstleistungen (530B), Universität Hohenheim

Fruwirthstraße 48, 70599 Stuttgart,

Deutschland

sodienst@uni-hohenheim.de

Danksagung. Die Autoren danken Herrn Professor Dr. Stenzl und Privatdozent Dr. Rausch vom Universitätsklinikum Tübingen für die Zusammenarbeit und Hilfe bei der Erstellung des Manuskriptes.

\section{Einhaltung ethischer Richtlinien}

Interessenkonflikt. H.Dick, S. Doth, C. Ernst, S. Fischer und M. Holderried geben an, dass kein Interessenkonflikt besteht.

Für diesen Beitrag wurden von den Autoren keine Studien an Menschen oder Tieren durchgeführt. Für die aufgeführten Studien gelten die jeweils dort angegebenen ethischen Richtlinien.

\section{Literatur}

1. Assadi M, Velez E, Najafi MH et al (2019) The need for standardization of nuclear cardiology reporting and data system (NCAD-RADS): learning from coronary artery disease (CAD), breast imaging (BI), liver imaging (LI), and prostate imaging (PI) RADS. J Nucl Cardiol 26:660-665

2. Behar BI, Guth C, Salfeld R (2016) Qualität im Krankenhaus - was Heilungserfolg und wirtschaftlichen Erfolg verbindet. In: Behar Bl, Guth C, Salfeld R (Hrsg) Modernes Krankenhausmanagement. Gabler, Wiesbaden, S219-234

3. Bostrom PJ, Toren PJ, Xi H et al (2011) Point-of-care clinical documentation: assessment of a bladder cancer informatics tool (eCancerCareBladder): a randomized controlled study of efficacy, efficiency and user friendliness compared with standard electronic medical records. J Am Med Inform Assoc 18:835-841

4. Breyer F, Zweifel P, Kifmann M (2013) Gesundheitsökonomik. Springer, Berlin, Heidelberg, New York

5. Bunck AC, Baessler B, Ritter C et al (2020) Structured reporting in cross-sectional imaging of the heart: reporting templates for CMR 
imaging of cardiomyopathies (myocarditis, dilated cardiomyopathy, hypertrophic cardiomyopathy, arrhythmogenic right ventricular cardiomyopathy and siderosis). Rofo 192:27-37

6. Chu S, Boxer R, Madison P et al (2015) Veterans affairs telemedicine: bringing urologic care to remote clinics. Urology 86:255-260

7. Ernst BP, Katzer F, Künzel J et al (2019) Impact of structured reporting on developing head and neck ultrasound skills. BMCMed Educ 19:102

8. Gesundheitswesen SZBDEI (2021) Digitalisierung für Gesundheit Ziele und Rahmenbedingungen eines dynamisch lernenden Gesundheitssystems. Gutachten 2021

9. Hamm B, Asbach P (2018) Magnetic resonance imaging of the prostate in the PI-RADS era. In: Hodler J, Kubik-Huch RA, von Schulthess GK (Hrsg) Diseases of the abdomen and pelvis 2018-2021: diagnosticimaging-IDKD book. Springer, Cham, $S$ 99-115

10. Holderried M, Ernst C, Holderried F et al (2017) The potential of eHealth in otorhinolaryngology-head and neck surgery: patients' perspectives. Eur Arch Otorhinolaryngol 274:2933-2943

11. Holderried M, Hoeper A, Holderried F et al (2021) Attitude and potential benefits of modern information and communication technology use and telemedicine in cross-sectoral solid organ transplant care. Sci Rep 11:9037

12. Holten-Rossing $H$, Larsen LG, Toft BG et al (2016) Consultation on urological specimens from referred cancer patients using real-time digital microscopy: optimizing the workflow. J Pathol Inform 7:11

13. Hunink MGM, Weinstein MC, Wittenberg $E$ et al (2014) Decision making in health and medicine: integrating evidence and values. Cambridge University Press, Cambridge

14. Jochimsen B (2021) Digitalisierung für Gesundheit - ökonomische Aspekte des Gutachtens des SVR Gesundheit. Wirtschaftsdienst 101:376-380

15. Jones $G$, Brennan V, Jacques $R$ et al (2018) Evaluating the impact of a 'virtual clinic' on patient experience, personal and provider costs of care in urinary incontinence: a randomised controlled trial. PLOSONE 13:e189174

16. Magnetta MJ, Donovan AL, Jacobs BL et al (2018) Evidence-based reporting: a method to optimize prostate MRI communications with referring physicians. AJRAm J Roentgenol 210:108-112

17. Marcovici PA, Taylor GA (2014) Journal club: structured radiology reports are more complete and more effective than unstructured reports. AJR Am JRoentgenol 203:1265-1271

18. Mata C, Walker PM, Oliver A et al (2016) ProstateAnalyzer: web-based medical application for the management of prostate cancer using multiparametric MR imaging. Inform Health Soc Care 41:286-306

19. Mimra W, Rasch A, Waibel C (2016) Price competition and reputation in credence goods markets: experimental evidence. Games Econ Behav 100:337-352

20. Mumm JN, Rodler S, Mumm ML et al (2020) Digitale Innovation in der Medizin - die COVID19-Pandemie als Akzelerator von "digital health". JUrol Urogynäkol 28:1-5

21. Novara G, Checcucci E, Crestani A et al (2020) Telehealth in urology: a systematic review of the literature. How much can telemedicine be useful during and after the COVID-19 pandemic? Eur Urol 78:786-811

\section{Current developments on digitalization. Analysis of quality and economics in healthcare}

Background: In the German healthcare system and thus also in the field of urology, economic conditions are becoming increasingly relevant and, in addition, digital applications are becoming more widely used.

Objectives: Health economic analysis of the framework of digitalization in the German healthcare system and selected areas of application in urology.

Methods: Analysis of the report of the German Advisory Council for the development of healthcare. Conduction of a systematic literature analysis on the use of structured reporting and analysis of selected literature on telemedical applications in urology from a health economic point of view.

Results: The German Advisory Council for the development of healthcare identifies the regulation and complexity of the German healthcare system as well as the handling of data protection and data security as key obstacles to digitalization. The use of structured reporting can increase the quality, effectiveness, and efficiency of reporting in urology. In terms of costs, significant savings can be realized with increasing digitalization in medicine.

Conclusions: From a medical and health economic perspective, there is a need for further development in the framework for digital applications in the German healthcare system with regard to information security and data protection. With the appropriate use of digital applications such as structured reporting and telemedicine, optimal conditions can be established for the increasing use of artificial intelligence in the field of urology.

\section{Keywords}

EHealth · Health economics · Telemedicine - Electronic health records · QALY

22. Pinto Dos Santos D, Baessler B (2018) Big data, artificial intelligence, and structured reporting. Eur Radiol Exp 2:42

23. Prütz F (2012) Was ist Qualität im Gesundheitswesen? Ethik Med 24:105-115

24. Röthke $M$, Blondin D, Schlemmer $\mathrm{H}-\mathrm{P}$ et al (2013) PI-RADS-Klassifikation: Strukturiertes Befundungsschema für die MRT der Prostata. Rofo 185:253-261

25. Salem J, Struck JP (2019) Stellenwert der Digitalisierung im urologischen Alltag. Aktuelle Urol 50:386-391

26. Shaish $\mathrm{H}$ (2020) Structured prostate MRI reporting: how and why. Abdom Radiol (NY) 45:3969-3973

27. Shaish H, Feltus W, Steinman J et al (2018) Impact of a structured reporting template on adherence to prostate imaging reporting and data system version 2 and on the diagnostic performance of prostate MRI for clinically significant prostate cancer. J Am Coll Radiol 15:749-754

28. Sosnowski R, Kamecki H, Joniau S et al (2020) Introduction of telemedicine during the COVID-19 pandemic: a challenge for now, an opportunity for the future. Eur Urol 78:820-821

29. Struck JP, Wenzel M, Heidenreich A et al (2018) Stellenwert der Digitalisierung im urologischen Alltag. Aktuelle Urol 49:509-514

30. Urbach N (2017) Betriebswirtschaftliche Besonderheiten digitaler Güter. In: 9. Forum für Verbraucherwissenschaft

31. Viers BR, Lightner DJ, Rivera ME et al (2015) Efficiency, satisfaction, and costs for remote video visits following radical prostatectomy: a randomized controlled trial. Eur Urol 68:729-735

32. Wenzel M, Salem J, Heidenreich A et al (2019) Telemedizin im urologischen Setting. Uro-News 23:38-40
33. Wetterauer C, Winkel DJ, Federer-Gsponer JR et al (2019) Structured reporting of prostate magnetic resonance imaging has the potential to improve interdisciplinary communication. PLOS ONE 14:e212444

34. Wetterauer C, Winkel DJ, Federer-Gsponer JR et al (2020) Novices in MRI-targeted prostate biopsy benefit from structured reporting of MRI findings. World JUrol 38:1729-1734

35. Zholudev V, Safir IJ, Painter MN et al (2018) Comparative cost analysis: teleurology vs conventional face-to-face clinics. Urology 113:40-44 